\title{
Using Multilayered Substrate Integrated Waveguide to Design Microwave Gain Equalizer
}

\author{
Yongfei Wang, ${ }^{1}$ Dongfang Zhou,, ${ }^{1}$ Yi Zhang, ${ }^{1,2}$ and Chaowen Chang ${ }^{1}$ \\ ${ }^{1}$ Department of Electromagnetic Wave and Antenna Propagation, Institute of Information Science and Technology of Zhengzhou, \\ Zhengzhou, Henan 450001, China \\ ${ }^{2}$ Microwave Tech and Antenna, Department of Electronic Engineering, Tsinghua University, Beijing 100084, China
}

Correspondence should be addressed to Yongfei Wang; zretaw0303@gmail.com

Received 8 February 2014; Revised 27 April 2014; Accepted 28 May 2014; Published 10 July 2014

Academic Editor: Yaseen Iqbal

Copyright (c) 2014 Yongfei Wang et al. This is an open access article distributed under the Creative Commons Attribution License, which permits unrestricted use, distribution, and reproduction in any medium, provided the original work is properly cited.

\begin{abstract}
This paper presents the design and experiment of a novel microwave gain equalizer based on the substrate integrated waveguide (SIW) technique. The proposed equalizer is formed by an SIW loaded by SIW resonators, which has very compact structure and can compensate for gain slope of microwave systems. Equivalent circuit analysis is given about the proposed structure for a better insight into the structure's response. A Ku-Band equalizer with four SIW resonators is simulated and fabricated with a multilayer printed circuit board process. The measured results show good performance and agreement with the simulated results; an attenuation slope of $-4.5 \mathrm{~dB}$ over $12.5-13.5 \mathrm{GHz}$ is reached with a size reduction of $76 \%$.
\end{abstract}

\section{Introduction}

Gain equalizers, a kind of microwave component with an attenuation characteristic that varies as a function of frequency, have been used as a means of gain slope equalization of microwave and millimeter wave systems for many years [1-4]. Equalizers in rectangular waveguide and microstrip type have found their applications in many microwave and millimeter systems. In [1], the design method for high power microwave gain equalizer of waveguide type used for travelling wave tube amplifiers (TWTAs) is presented. In [2] a microstrip-type equalizer by stepped impedance resonator with thin film resistance is reported. In [3] a broadband amplifier gain equalizer with a temperature variable attenuator and a temperature variable network to compensate for the gain slope variation versus frequency and temperature is designed. Active equalizer of microstrip type using $\mathrm{p}-\mathrm{i}-$ $\mathrm{n}$ diodes has been reported [4]. However, due to the threedimensional (3D) nature of rectangular waveguides, it can be difficult to minimize in size and integrate with other equipment. The low $Q$-factor with high insertion loss of microstrip has made it impossible to fulfill the extreme needs of high power amplifiers.
Substrate integrated waveguide (SIW) is a new planar electromagnetic guide wave structure. It takes the advantages of low loss, low cost, simple integration with planar devices, a size reduction of a factor $\varepsilon_{r}^{-1 / 2}[5]$, and possibility of easy integration into microwave and millimeter wave integrated circuits. Compared with microstrip-like structures, it has higher $Q$-factor [6-8]. In view of the above advantages, it has found its application in antennas, couplers, and filters [9-12]. It is a fact that resonator shows bandstop characteristic within a narrow band. Then a resonator with comparable attenuation can serve as gain adjustment equipment in a relative narrow band, and cascaded resonators with attenuation characteristic varying as a function of frequency can serve as gain equalizer. For the first time, this paper presents a design of a multilayered substrate integrated waveguide equalizer using E-plane coupling SIW resonators. Exact resonance will be obtained by changing the coupling, and shift of the excitation resulting in change of the resonance frequency could be used as a tuning mechanism of the resonator. We can also use the H-plane coupling structure of single layer to design the equalizer. However, the multilayer topology allows various coupling mechanisms, and the arrangement of the resonators becomes 
more flexible. Besides, the E-plane coupling resonators can further reduce the circuit size.

\section{Equivalent Circuit Analysis}

Figure 1(a) shows a kind of two-port resonant system; it is composed of a cavity coupled to a transmission line. This structure can be modeled as in Figure 1(b). The impedance $Z_{\text {in }}$ is the input impedance of the resonator:

$$
\begin{gathered}
Z_{\text {in }}=R\left(1+j 2 Q_{0} \frac{f-f_{0}}{f_{0}}\right), \\
\beta=\frac{n^{2} z_{0}}{2 R},
\end{gathered}
$$

where $Q_{0}$ is the unloaded quality factor of the resonator, $\beta$ is the coupling efficient, and $R$ is the equivalent resistance.

The resonator and the coupling system can be seen as a resistance $Z_{\text {in }}$ parallel with the transmission line (Figure 1(c)):

$$
Z=\frac{Z_{\text {in }}}{n^{2}}=\frac{Z_{0}}{2 \beta}\left(1+j 2 Q_{0} \frac{f-f_{0}}{f_{0}}\right) .
$$

Then normalize susceptance $Y=z_{0} / z$; the scattering parameters are

$$
\begin{aligned}
S_{11} & =\frac{-Y}{2+Y} \\
& =\frac{-1}{((1+\beta) / \beta)\left[1+j\left(2 Q_{0}\left(f-f_{0}\right) /(1+\beta) f_{0}\right)\right]}, \\
S_{21} & =\frac{2}{2+Y} \\
& =1+\frac{-1}{((1+\beta) / \beta)\left[1+j\left(2 Q_{0}\left(f-f_{0}\right) /(1+\beta) f_{0}\right)\right]} .
\end{aligned}
$$

It can be seen from (3) that the coupling efficient and unloaded quality factor are the key factors to determine the transmission characteristic of the cavity, once the resonant frequency is determined. The gain slope is the derivation of $|S 21|$. When

$$
f=\frac{6 Q_{0} \pm \sqrt{3 \sqrt{(\beta+1)^{2}+1}-3}}{6 Q_{0}} f_{0},
$$

the gain slope comes to its extreme, and the extreme points are symmetrical about $f_{0}$.

When using two or more cascaded resonators to realize an equalizer, the cascaded $S$ parameters can be calculated as [13]

$$
\left[S_{A B}\right]=\left[\begin{array}{cc}
S_{11 A}+\frac{S_{11 B} S_{12 A} S_{21 A}}{1-S_{11 B} S_{22 A}} & \frac{S_{12 A} S_{12 B}}{1-S_{11 B} S_{22 A}} \\
\frac{S_{21 A} S_{21 B}}{1-S_{11 B} S_{22 A}} & S_{22 B}+\frac{S_{22 A} S_{12 B} S_{21 B}}{1-S_{11 B} S_{22 A}}
\end{array}\right] .
$$

\section{Resonator and Its Coupling Structure Design}

Figure 2 shows the schematic of one E-plane coupling SIW resonator. The SIW main transmission structure consists of the substrate at the bottom, and the SIW resonator consists of the substrate at the top. The equivalent waveguide sidewalls are synthesized by a group of metalized vias in a PCB or LTCC process. The cavity is excited by a coupling probe at the common broad wall which is used to couple energy in the SIW main transmission structure into the resonator, with a circular slot around to separate it from the SIW inner metal plane. Therefore, one circular slot is created around the coupling probe. The substrate of the two layers can be chosen according to the design. Here the structure is implemented on two-layer RT/Duroid 5880 substrate with dielectric constant 2.2 and loss tangent 0.0009 . Both of the layers have a thickness of $0.508 \mathrm{~mm}$. The layer of Taconic TPG-30 material is used as a bonding layer to combine the two layers of substrate, which is $0.12 \mathrm{~mm}$ thick with dielectric constant 3.0 and loss tangent 0.0038 .

To design SIW devices, the width of SIW properly operating in the same frequency range should be determined at first [8]:

$$
w_{\text {eff }}=W_{1}-\frac{4 r^{2}}{0.95 s}
$$

where $W_{1}$ is the transverse distance between two rows of vias, which is also the width of SIW cavity, seen from Figure 4(a), $r$ is the radius of the metal vias, and $s$ represents the longitudinal distance. The cutoff frequency of the SIW is determined by [14]

$$
f_{c}=\frac{c}{2 \sqrt{\varepsilon_{r}}}\left(W_{1}-\frac{4 r^{2}}{0.95 s}\right)^{-1},
$$

where $c$ is light velocity in vacuum and $\varepsilon_{r}$ is the relative dielectric permittivity.

Experiments in [7] show that the field distribution of TE10-like mode in the SIW is very similar to the mode in a conventional rectangular waveguide. Thus, SIW cavity can be designed by using the equations [12]

$$
\begin{gathered}
f_{0}=\frac{c}{2 \sqrt{\varepsilon_{r}}} \sqrt{\frac{1}{L_{\mathrm{eff}}^{2}}+\frac{1}{W_{\mathrm{eff}}^{2}}}, \\
L_{\mathrm{eff}}=L_{1}-\frac{4 r^{2}}{0.95 s},
\end{gathered}
$$

where $L_{1}$ is the length of SIW cavity.

To simplify the design procedure, the SIW cavity dimensions are selected to be an integer multiple of the space between metallic vias.

The principle of modifying the E-field and $\mathrm{H}$-fields distributions through local perturbations is used to design the equalizer. When the cavity dimensions are determined, by placing a probe together with a slot as a coupling structure, 


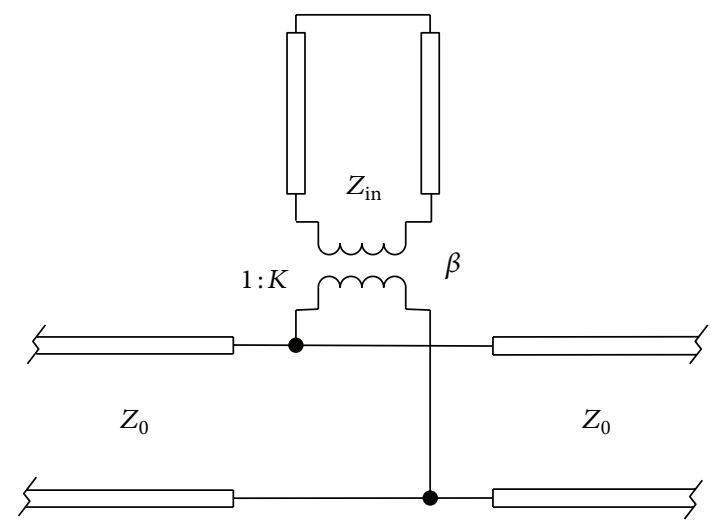

(a)

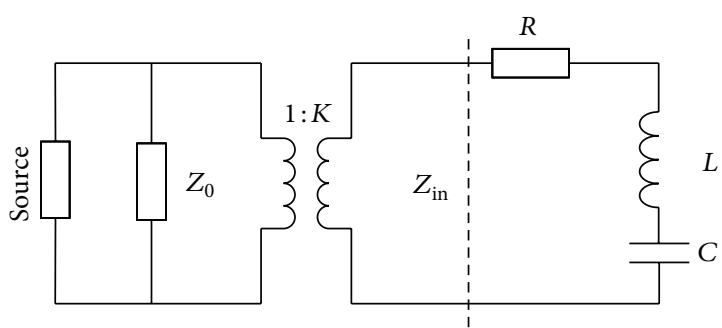

(b)

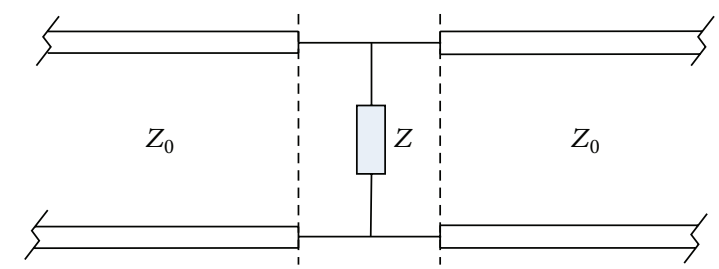

(c)

Figure 1: Schematic of a two-port resonant system and its equivalent circuit. (a) Two-port resonant system. (b) Series resonant equivalent circuit with coupling. (c) Using resistance $Z$ to equate the resonator and coupling structure.

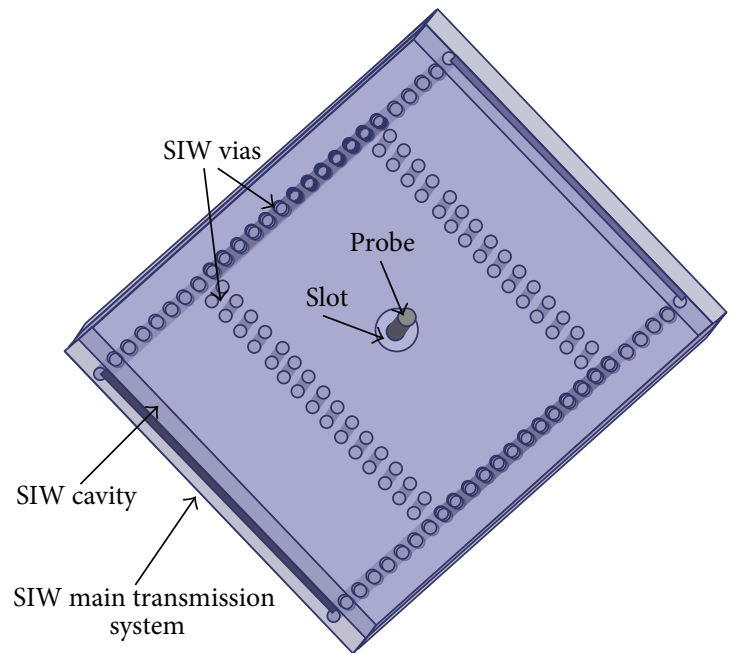

(a)

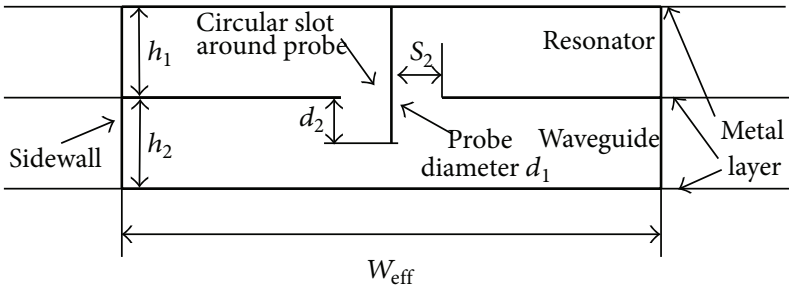

(b)

FIgURE 2: Schematic of the proposed SIW resonator.

fields' distribution in the cavity has changed. Hence the resonant frequency will change as in [15]:

$$
\frac{f-f_{0}}{f_{0}}=\frac{\Delta W_{m}-\Delta W_{e}}{W_{m}+W_{e}},
$$

where $f$ is resonant frequency of the cavity after perturbation and $f_{0}$ is the original resonant frequency of the original cavity. $W_{m}$ and $W_{e}$ are the stored magnetic and electric energy in the cavity, and $\Delta W_{m}$ and $\Delta W_{e}$ are the changes of magnetic and electric energy, respectively, of the cavity with coupling structures.

For a traditional rectangular waveguide excited by a probe, it is preferred that the existence of the probe will not disturb the fields in the structure. In the case of SIW structures, due to the very thin substrate, the probe will inevitably change the fields [15]. So the depth of probe inserting to the resonator ought to be optimized carefully. The simulations show that the change of insertion depth brings very small change in the resonant frequency with great 


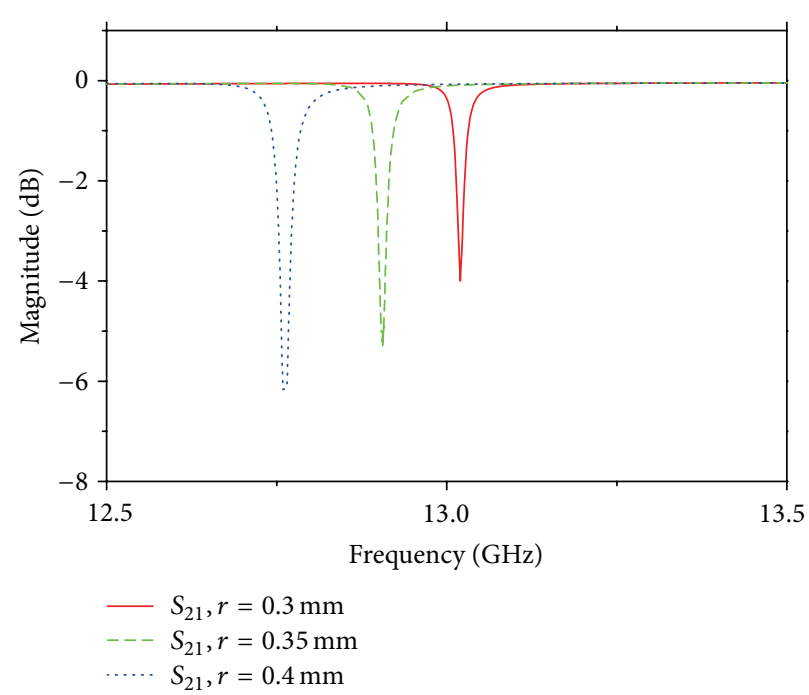

(a)

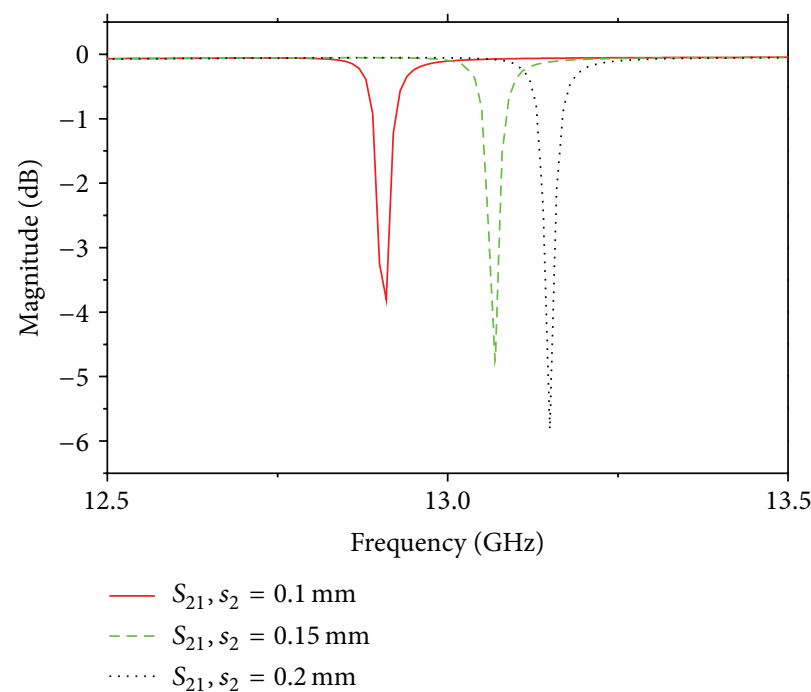

(b)

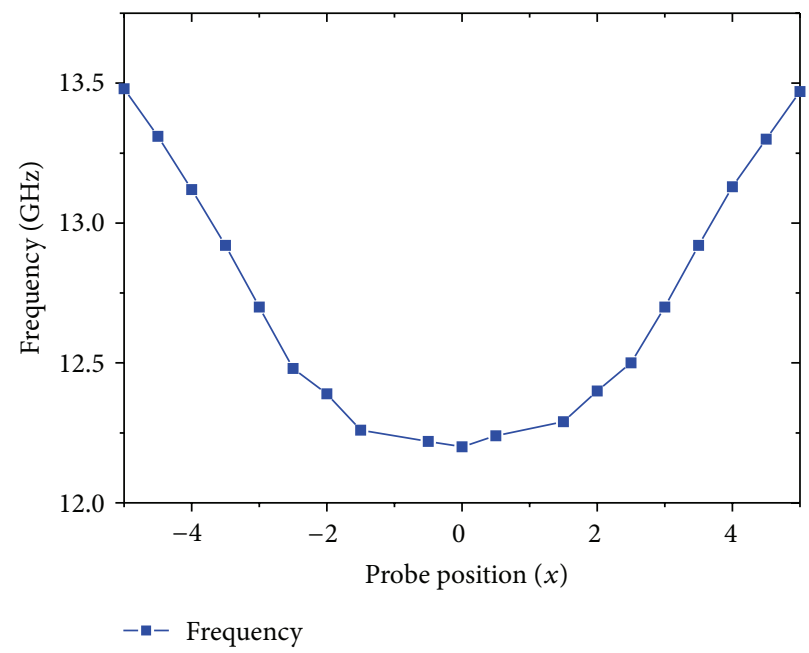

(c)

FIGURE 3: Simulation results of the proposed SIW resonator. (a) Change of probe radius when slot width at the center of the cavity is 0.05 mm. (b) Change of slot width while probe is at the center with its radius $0.3 \mathrm{~mm}$. (c) Change of probe position along $x$-axis with its radius being $0.3 \mathrm{~mm}$ and annular slot width being $0.05 \mathrm{~mm}$.

change in insertion loss. The simulation results are omitted here for brevity. To simplify the fabrication procedure, the insertion depth is selected to be an integer multiple of the TPG-30 substrate, and here it is $0.12 \mathrm{~mm}$. The coupling probe is isolated from the SIW lower conductor planes through an annular slot that results in a circular disk around the coupling probe. The magnitude of electric field comes to its maximum at the edge of the slot. Any change in this area has negligible influence on the fields' distribution, resulting in change of resonant frequency and insertion loss. Figures 3(a) and 3(b) illustrate the simulation results of $S_{21}$-parameters for different probe radii and slot widths. It can be seen that resonant frequency increases slightly by increasing the slot width or decreasing the probe radius. Also, the insertion loss at resonant frequency has changed noticeably in all cases. It is clear that the dominant factor to determine the resonant frequency is resonator dimensions. The place and the width of probe and slot can also influence the resonant frequency and its insertion loss. Thus the radius and the slot width together with its position can all be used to tune the coupling. Figure 3(c) shows the change of resonant frequency as a function of the change of the probe position along $x$-axis.

It can be seen from Figure 3 that the gain slope of the lower band from central frequency of each resonator is negative and that of the upper band is positive. When two or more resonators of such kind with different resonant frequency are cascaded together, the gain characteristic shows a curve with different slopes at different frequencies. 


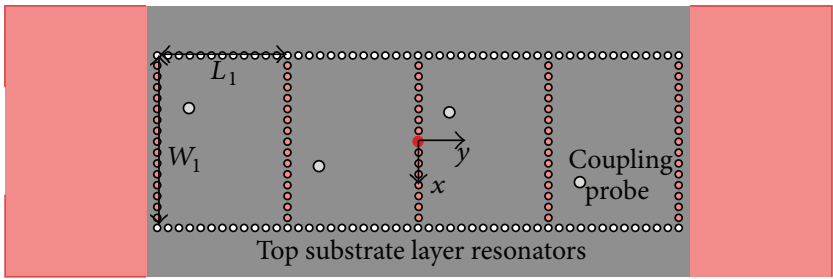

(a)

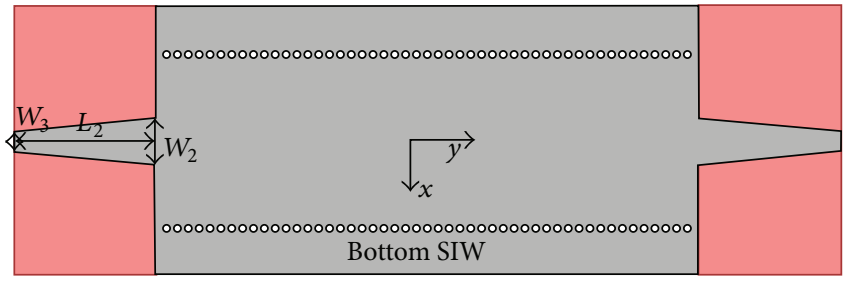

(c)

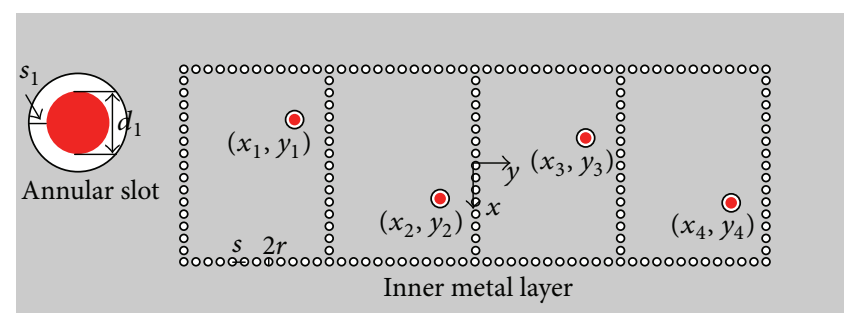

(b)

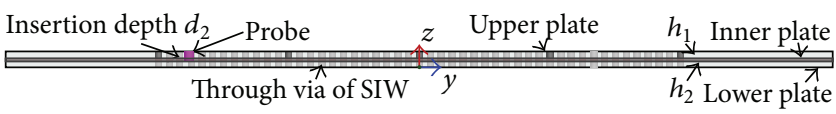

(d)

Figure 4: Configuration of the proposed equalizer. (a) Top view. (b) Inner view. (c) Bottom view. (d) Side view.

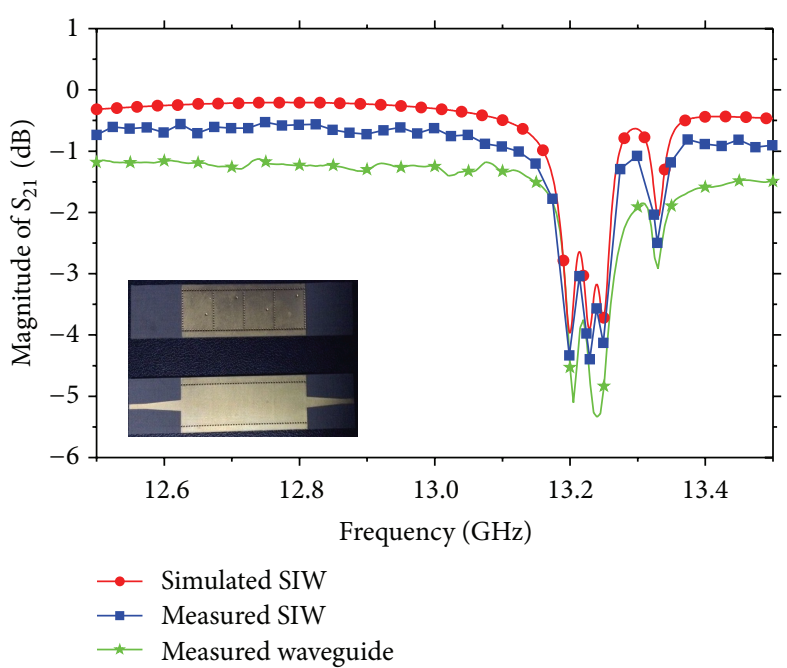

(a)

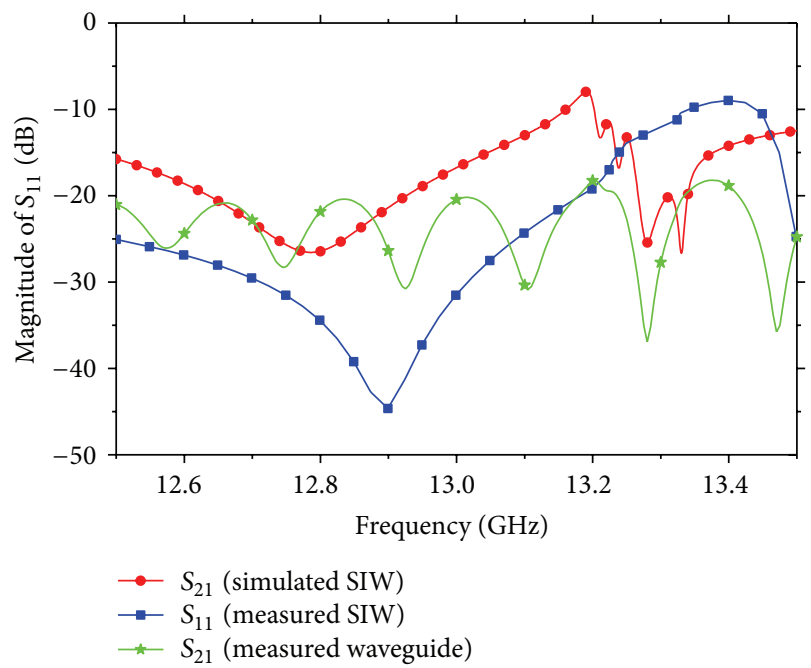

(b)

FIGURE 5: $S$-parameter against operational frequency. (a) Measured and simulated $S_{21}$-parameter for proposed SIW and rectangular waveguide equalizer. (b) Measured and simulated $S_{11}$-parameter for proposed SIW and rectangular waveguide equalizer.

\section{Proposed Equalizer and Experimental Results}

We consider the proposed E-plane coupling SIW equalizer as shown in Figure 4. The proposed equalizer is mainly composed of three parts: one SIW-based rectangular waveguide, four SIW-based E-plane coupling resonators, and two microstrip-to-SIW transitions for measurement. The parameter values are listed in Table 1.

To verify the performance, a Ku-Band equalizer with four SIW resonators is designed and fabricated using multilayered PCB process. The equalizer is measured using an Agilent N5244A network analyzer with coaxial calibration. Figure 5 shows the measured and simulated results of the equalizer.
We note that the maximum insertion loss is approximately $0.6 \mathrm{~dB}$ with a return loss better than $8.5 \mathrm{~dB}$. Adapters and SMA connectors are not deembedded from the measurement and therefore contribute to the insertion and return loss. Figure 5 also shows the measured results of a rectangular waveguide equalizer with isolators at each port. This equalizer also utilizes four resonators resonating at the same frequencies as that of SIW equalizer; its maximum insertion loss is $1.2 \mathrm{~dB}$ with a return loss better than $18.2 \mathrm{~dB}$. Due to the effect of isolators, better return loss can be reached for the rectangular waveguide equalizer.

The proposed equalizers are evaluated with a TWTA whose ideal gain is $42 \mathrm{~dB}$ over its working band of 12.513.5 GHz. In fact, it has a gain of $42 \mathrm{~dB}$ with a maximum 
TABLE 1: Design parameter values of proposed equalizer.

\begin{tabular}{lcccccccccccccccccccc}
\hline Parameter & $W_{1}$ & $L_{1}$ & $W_{2}$ & $W_{3}$ & $L_{2}$ & $r$ & $s$ & $d_{1}$ & $s_{1}$ & $x_{1}$ & $y_{1}$ & $x_{2}$ & $y_{2}$ & $x_{3}$ & $y_{3}$ & $x_{4}$ & $y_{4}$ & $h_{1}$ & $h_{2}$ & $d_{2}$ \\
\hline Value $(\mathrm{mm})$ & 6.4 & 9.6 & 1.78 & 0.76 & 11.2 & 0.25 & 0.8 & 0.8 & 0.1 & -2.5 & -16.9 & 1.8 & -7.3 & -2.2 & 2.3 & 3 & -11.9 & 0.508 & 0.628 & 0.12 \\
\hline
\end{tabular}

gain slope of $5 \mathrm{~dB}$ over the working band. By using the SIW equalizer, the gain slope has been improved to $0.5 \mathrm{~dB}$.

\section{Conclusion}

An equalizer using multilayered SIW technique is proposed in this paper. A Ku-Band equalizer using the proposed technique has been designed, fabricated, and measured. The simulated and measured results are in good agreement. The proposed equalizer has good performance and small size and can be directly integrated with other planar circuits without any additional mechanical assembling tuning. The developed SIW equalizer can serve as a building block in the design of small size, highly integrated microwave and millimeter wave TWTA.

\section{Conflict of Interests}

The authors declare that there is no conflict of interests regarding the publication of this paper.

\section{Acknowledgments}

The authors would like to thank W. Hong for his assistance in the fabrication of the MSIWs. This work was supported in part by the Chinese Ministry of Industry and Information Technology and Chinese Ministry of Science under Grant 2013-ZX01010001-003.

\section{References}

[1] D. F. Zhou, G. X. Sun, Z. X. Niu, J. P Ren, and C. Lv, “The analyzing and optimization designing of high-power microwave equalizer," Vacuum Electronics, vol. 2, pp. 1-10, 2000.

[2] T. Zhou, Z. Wang, W. Huan, and R. Xu, "Design of microwave wave gain equalizer using microstrip shorted SIR," in Proceedings of the International Conference on Microwave and Millimeter Wave Technology (ICMMT '12), vol. 5, pp. 1-4, IEEE, 2012.

[3] A. Fejzuli, R. Kaarsberg, and N. Roldan, "Broadband amplifier gain slope equalization with a single passive component," High Frequency Electronics, vol. 5, no. 6, pp. 22-26, 2006.

[4] S. C. Bera, "Amplitude tilt active equalizer for frequency and temperature compensation," IEEE Microwave and Wireless Components Letters, vol. 21, no. 7, pp. 344-346, 2011.

[5] N. Grigoropoulos, B. Sanz-Izquierdo, and P. R. Young, "Substrate integrated folded waveguides (SIFW) and filters," IEEE Microwave and Wireless Components Letters, vol. 15, no. 12, pp. 829-831, 2005.

[6] D. Deslandes and K. Wu, "Integrated microstrip and rectangular waveguide in planar form," IEEE Microwave and Wireless Components Letters, vol. 11, no. 2, pp. 68-70, 2001.
[7] F. Xu, Y.Zhang, W. Hong, K. Wu, and T. J. Cui, "Finite-difference frequency-domain algorithm for modeling guided-wave properties of substrate integrated waveguide," IEEE Transactions on Microwave Theory and Techniques, vol. 51, no. 11, pp. 2221-2227, 2003.

[8] M. Bozzi, A. Georgiadis, and K. Wu, "Review of substrateintegrated waveguide circuits and antennas," IET Microwaves, Antennas \& Propagation, vol. 5, no. 8, pp. 909-920, 2011.

[9] Y. S. Zhang and W. Hong, "A millimeter-wave gain enhanced multi-beam antenna based on a coplanar cylindrical dielectric lens," IEEE Transactions on Antennas and Propagation, vol. 60, no. 7, pp. 3485-3488, 2012.

[10] K. Gong, W. Hong, Y. Zhang, P. Chen, and C. J. You, "Substrate integrated waveguide quasi-elliptic filters with controllable electric and magnetic mixed coupling," IEEE Transactions on Microwave Theory and Techniques, vol. 60, no. 10, pp. 3071-3078, 2012.

[11] D. F. Guan, Z.-P. Qian, Y.-S. Zhang, Y. Cai, and W.-Q. Cao, "Hybrid SIW-GCPW narrow-wall $3 \mathrm{~dB}$ coupler," Frequenz, vol. 67, no. 7-8, pp. 209-212, 2013.

[12] J. D. Barrera and G. H. Huff, "Analysis of a variable SIW resonator enabled by dielectric material perturbations and applications," IEEE Transactions on Microwave Theory and Techniques, vol. 61, no. 1, pp. 225-233, 2013.

[13] D. M. Pozar, Microwave Engineering: Fourth Edition, John Wiley \& Sons, New York, NY, USA, 2011.

[14] Y. Cassivi, L. Perregrini, P. Arcioni, M. Bressan, K. Wu, and G. Conciauro, "Dispersion characteristics of substrate integrated rectangular waveguide," IEEE Microwave and Wireless Components Letters, vol. 12, no. 9, pp. 333-335, 2002.

[15] F. T. Ladani, S. Jam, and R. Safian, "A new SIW resonator excitation method and its equivalent circuit," Microwave and Optical Technology Letters, vol. 55, no. 5, pp. 1133-1138, 2013. 

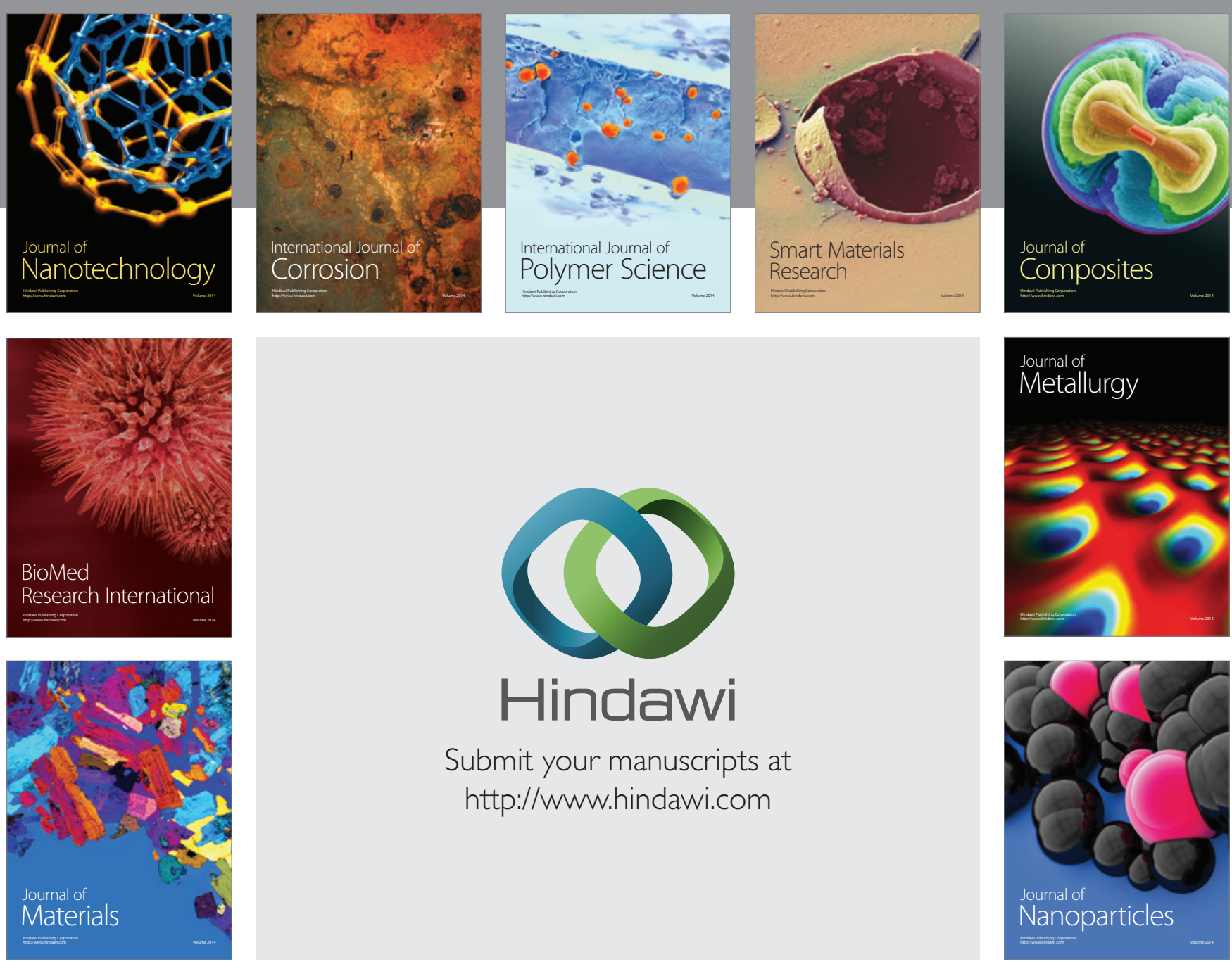

Submit your manuscripts at http://www.hindawi.com
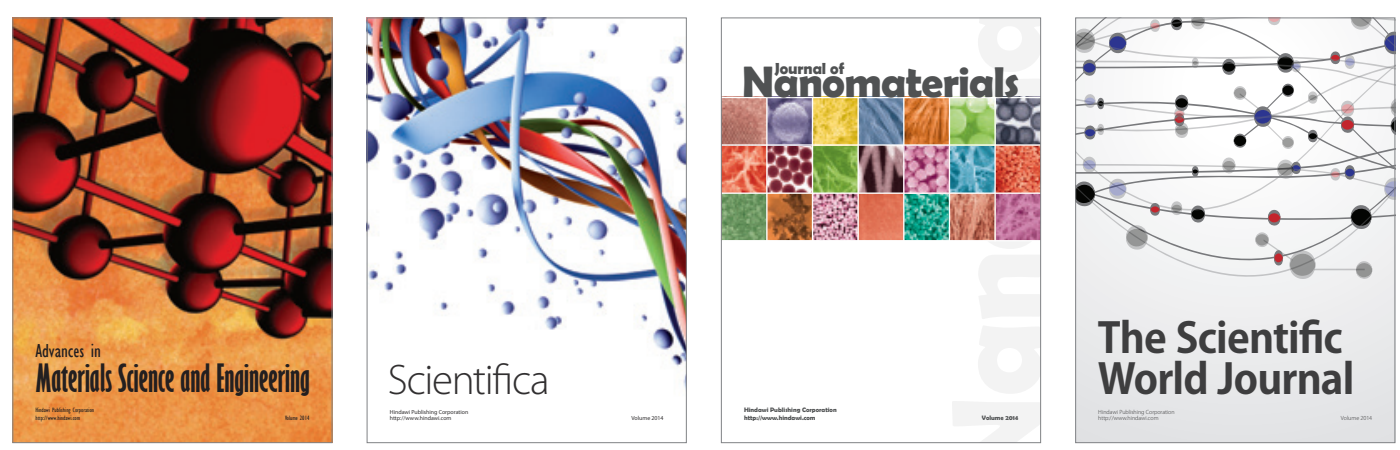

\section{The Scientific World Journal}
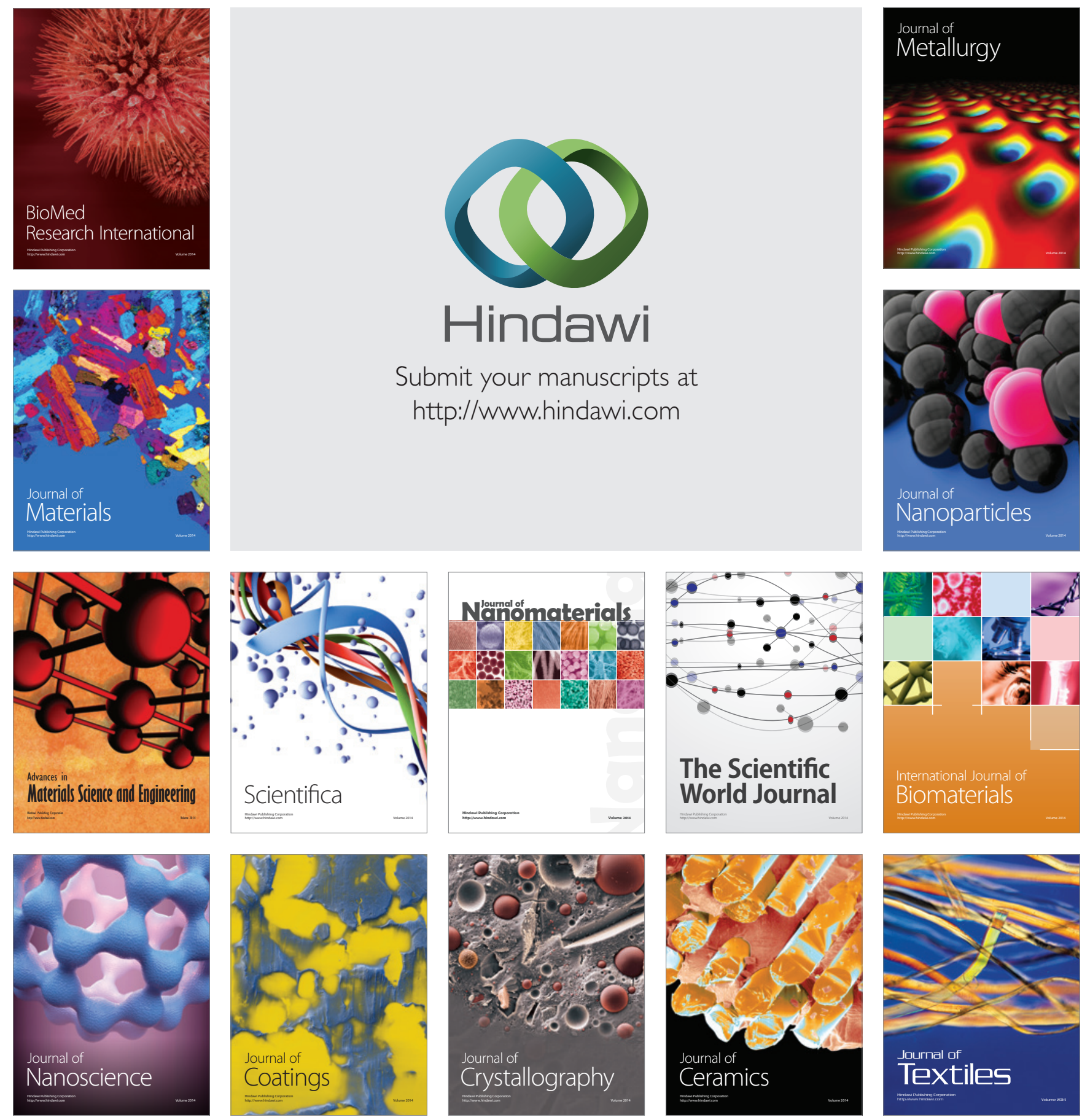